\title{
On the Complexity of the $k$-Level in Arrangements of Pseudoplanes
}

\author{
Micha Sharir \\ School of Computer Science, Tel Aviv University, Tel Aviv, Israel \\ http://www.cs.tau.ac.il/ michas/ \\ michas@post.tau.ac.il
}

Chen Ziv

School of Computer Science, Tel Aviv University, Tel Aviv, Israel

henziv@gmail.com

\begin{abstract}
A classical open problem in combinatorial geometry is to obtain tight asymptotic bounds on the maximum number of $k$-level vertices in an arrangement of $n$ hyperplanes in $\mathbb{R}^{d}$ (vertices with exactly $k$ of the hyperplanes passing below them). This is essentially a dual version of the $k$-set problem, which, in a primal setting, seeks bounds for the maximum number of $k$-sets determined by $n$ points in $\mathbb{R}^{d}$, where a $k$-set is a subset of size $k$ that can be separated from its complement by a hyperplane. The $k$-set problem is still wide open even in the plane. In three dimensions, the best known upper and lower bounds are, respectively, $O\left(n k^{3 / 2}\right)[15]$ and $n k \cdot 2^{\Omega(\sqrt{\log k})}[19]$.

In its dual version, the problem can be generalized by replacing hyperplanes by other families of surfaces (or curves in the planes). Reasonably sharp bounds have been obtained for curves in the plane $[16,18]$, but the known upper bounds are rather weak for more general surfaces, already in three dimensions, except for the case of triangles [1]. The best known general bound, due to Chan [7] is $O\left(n^{2.997}\right)$, for families of surfaces that satisfy certain (fairly weak) properties.

In this paper we consider the case of pseudoplanes in $\mathbb{R}^{3}$ (defined in detail in the introduction), and establish the upper bound $O\left(n k^{5 / 3}\right)$ for the number of $k$-level vertices in an arrangement of $n$ pseudoplanes. The bound is obtained by establishing suitable (and nontrivial) extensions of dual versions of classical tools that have been used in studying the primal $k$-set problem, such as the Lovász Lemma and the Crossing Lemma.
\end{abstract}

2012 ACM Subject Classification Mathematics of computing $\rightarrow$ Combinatorics; Theory of computation $\rightarrow$ Computational geometry

Keywords and phrases k-level, pseudoplanes, arrangements, three dimensions, k-sets

Digital Object Identifier 10.4230/LIPIcs.SoCG.2019.62

Related Version A full version of this paper is available at https://arxiv.org/abs/1903.07196.

Funding Work on this paper was supported by Grants 892/13 and 260/18 from the Israel Science Foundation.

Micha Sharir: Work on this paper was also supported by Grant G-1367-407.6/2016 from the German-Israeli Foundation for Scientific Research and Development, by the Blavatnik Research Fund in Computer Science at Tel Aviv University, and by the Israeli Centers of Research Excellence (I-CORE) program (Center No. 4/11).

\section{Introduction}

Let $\Lambda$ be a set of $n$ non-vertical planes (resp., pseudoplanes, as will be formally defined shortly) in $\mathbb{R}^{3}$, in general position. We say that a point $p$ lies at level $k$ of the arrangement $\mathcal{A}(\Lambda)$, and write $\lambda(p)=k$, if exactly $k$ planes (resp., pseudoplanes) of $\Lambda$ pass below $p$. The $k$-level of $\mathcal{A}(\Lambda)$ is the closure of the set of points that lie on the planes of $\Lambda$ and are at level $k$. Our goal is to obtain an upper bound on the complexity of the $k$-level of $\mathcal{A}(\Lambda)$, which is 
measured by the number of vertices of $\mathcal{A}(\Lambda)$ that lie at level $k$. (The level may also contain vertices at level $k-1$ or $k-2$, but we ignore this issue - it does not effect the worst-case asymptotic bound that we are after.) Using a standard duality transform that preserves the above/below relationship (see, e.g., [10]), the case of planes is the dual version of the following variant of the $k$-set problem: given a set of $n$ points in $\mathbb{R}^{3}$ in general position, how many triangles spanned by $P$ are such that the plane supporting the triangle has exactly $k$ points of $P$ below it? We refer to these triangles as $k$-triangles. This has been studied by Dey and Edelsbrunner [9], in 1994, for the case of halving triangles, namely $k$-triangles with $k=(n-3) / 2$ (and $n$ odd). They have shown that the number of halving triangles is $O\left(n^{8 / 3}\right)$. In 1998, Agarwal et al. [1] generalized this result for $k$-triangles, for arbitrary $k$, showing that their number is $O\left(n k^{5 / 3}\right)$, using a probabilistic argument. In 1999, Sharir, Smorodinsky and Tardos [15] improved the upper bound for the number of $k$-triangles in $S$ to $O\left(n k^{3 / 2}\right)$. Chan [6] has adapted a dualized view of the technique of Sharir et al. [15] in order to study the bichromatic $k$-set problem: given two sets $R$ and $B$ of points in $\mathbb{R}^{2}$ of total size $n$ and an integer $k$, how many subsets of the form $(R \cap h) \cup(B \backslash h)$ can have size exactly $k$ over all halfplanes $h$ ? This problem arises when we estimate the number of vertices at level $k$, in an arrangement of $n$ planes in 3 -space, that lie on one specific plane.

The three-dimensional case extends the more extensively studied planar case. In its primal setting, we have a set $S$ of $n$ points in the plane in general position, and a parameter $k<n$, and we seek bounds on the maximum number of $k$-edges, which are segments spanned by pairs of points of $S$ so that one of the halfplanes bounded by the line supporting the segment, say the lower halfplane, contains exactly $k$ points of $S$. In the dual version, we seek bounds on the maximum number of vertices of an arrangement of $n$ nonvertical lines in general position that lie at level $k$. The best known upper bound for this quantity, due to Dey [8], is $O\left(n k^{1 / 3}\right)$, and the best known lower bound, due to Tóth [19] is $n e^{\Omega(\sqrt{\ln k})}$ (Nivasch [13] has slightly improved this bound for the case of halving edges).

In this paper we consider the dual version of the problem in three dimensions, where the points are mapped to planes, and the $k$-triangles are mapped to vertices of the arrangement of these planes at level $k$. We translate parts of the machinery developed in [15] to the dual setting, and then extend it to handle the case of pseudoplanes. In the primal setting, we have a set $S$ of $n$ points in $\mathbb{R}^{3}$ in general position, and the set $T$ of $k$-triangles spanned by $S$. We say that triangle $\Delta_{1}$ crosses another triangle $\Delta_{2}$ if the triangles share exactly one vertex, and the edge opposite to that vertex in $\Delta_{1}$ intersects the interior of $\Delta_{2}$. Denote the number of ordered pairs of crossing $k$-triangles by $X^{k}$. The general technique in [15] is to establish an upper bound and a lower bound on $X^{k}$, and to combine these two bounds to derive an upper bound for the number of $k$-triangles in $S$.

The upper bound in [15] is based on the 3-dimensional version of the Lovász Lemma, as in [5]: Any line crosses at most $O\left(n^{2}\right)$ interiors of $k$-triangles. The lemma follows from the main property of the set $T$, which is its antipodality. Informally, the property asserts that for each pair of points $a, b \in S$, the $k$-triangles having $a b$ as an edge form an antipodal system, in the sense that for any pair $\Delta_{a b c}, \Delta_{a b d}$ of such triangles that are consecutive in the circular order around $a b$, the dihedral wedge that is formed by the two halfplanes that contain $\Delta_{a b c}, \Delta_{a b d}$, and are bounded by the line through $a b$, has the property that its antipodal wedge, formed by the two complementary halfplanes within the planes supporting $\Delta_{a b c}, \Delta_{a b d}$, contains a point $e \in S$ such that $\Delta_{a b e}$ is also a $k$-triangle; See Figure 1.

To obtain a lower bound on $X^{k}$, the technique in [15] defines, for each $a \in S$, a graph $G_{a}=\left(V_{a}, E_{a}\right)$ drawn in a horizontal plane $h_{a}^{+}$slightly above $a$, whose edges are, roughly, the cross-sections of the $k$-triangles incident to $a$ with the plane (see Figure 2). The analysis 


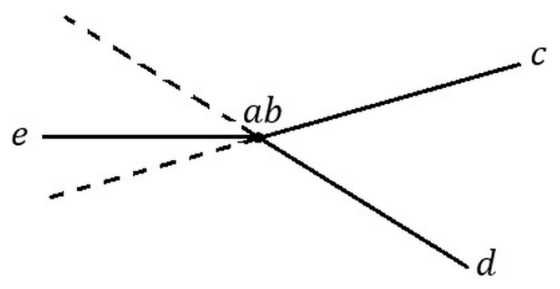

Figure 1 The antipodality property of $k$-triangles: the edge $a b$ is drawn from a side-view as a point. The triangles $\Delta_{a b c}, \Delta_{a b d}$ and $\Delta_{a b e}$, drawn as segments, are all $k$-triangles, where $e$ is contained in the antipodal wedge formed by the two complementary halfplanes within the planes supporting $\Delta_{a b c}, \Delta_{a b d}$.

in [15] shows that $G_{a}$ inherits the antipodality property of the $k$-triangles, and uses this fact to decompose $G_{a}$ into a collection of convex chains, and to estimate the number of crossings between the chains. Summing these bounds over all $a \in S$, the lower bound on $X^{k}$ follows.
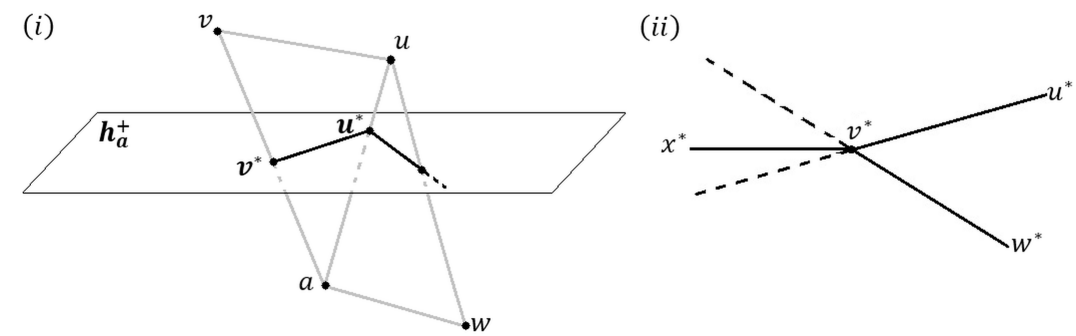

Figure 2 (i) The graph $G_{a}$ on $h_{a}^{+} . \Delta_{a u w}, \Delta_{a u v}$ are halving triangles. $u v$ is mapped to the segment $u^{*} v^{*}$, and $u w$ is mapped to a ray emanating from $u^{*}$ on $h_{a}^{+}$. (ii) The antipodality property in $G_{a}$.

We omit further details of the way in which these lower bounds are derived in [15], because, in the dual version that we present here, we use a weaker lower bound, which is based on a dual version of the Crossing Lemma (see [4]), and which is easier to extend to the case of pseudoplanes. Let $G=(V, E)$ be a simple graph, and define the crossing number of $G$ as the minimum number of intersecting pairs of edges in any drawing of $G$ in the plane. In the primal setting, the Crossing Lemma asserts that any simple graph $G=(V, E)$ drawn in the plane, with $|E|>4|V|$, has crossing number at least ${ }^{1} \frac{|E|^{3}}{64|V|^{2}}$. Using this technique for deriving a lower bound on $X^{k}$, instead of the refined technique in [15], one can show that the number of $k$-triangles is $O\left(n^{8 / 3}\right)$, or, with the additional technique of [1], $O\left(n k^{5 / 3}\right)$.

We now present the dual setting for the problem, where the input is a set $\Lambda$ of $n$ non-vertical planes in $\mathbb{R}^{3}$ in general position.

- Definition 1. Let $a, b, c \in \Lambda$. The open region between the lower envelope and the upper envelope of $a, b, c$ is called the corridor of $\boldsymbol{a}, \boldsymbol{b}, \boldsymbol{c}$ and is denoted by $\boldsymbol{C}_{\boldsymbol{a}, \boldsymbol{b}, \boldsymbol{c}}$.

The planes $a, b, c \in \Lambda$ divide the space into eight disjoint octant-like portions, and $C_{a, b, c}$ is the union of six of those portions, excluding the upper and the lower octants. We will be mostly interested in corridors $C_{a, b, c}$ for which the point $p_{a, b, c}=a \cap b \cap c$ (the unique vertex of $\left.C_{a, b, c}\right)$ is at level $k$. We will refer to such corridors as $k$-corridors, and define $C^{k}$ as the collection of $k$-corridors in $\mathcal{A}(\Lambda) ; k$-corridors serve as a dual version of $k$-triangles.

1 The constant of proportionality has been improved in subsequent works, but we will stick to this bound. 
- Definition 2. We say that a corridor $C_{1}$ is immersed in a corridor $C_{2}$ in $\mathcal{A}(\Lambda)$ if they share exactly one plane, and the intersection line of the other two planes of $C_{1}$ is fully contained in $C_{2}$. Let $X^{k}$ denote the number of ordered pairs of immersed corridors in $C^{k}$.

Immersion of $k$-corridors is the dual notion of crossings of $k$-triangles. Note that if a corridor $C_{1}$ is immersed in a corridor $C_{2}$, it cannot be that $C_{2}$ is also immersed in $C_{1}$.

In the full version of the paper [17] (see also [20]), we provide details of this dual setup. We define $X^{k}$ as the number of ordered pairs of immersed $k$-corridors, and present the derivation of the upper bound and the lower bound on $X^{k}$. In this abstract, we only consider in detail the extension to the case of pseudoplanes, which is our main topic of interest. We remark, though, that this translation to the dual context, although routine in principle, is rather involved and nontrivial, and requires careful handling of quite a few details.

\section{The case of pseudoplanes}

We say that a family $\Lambda$ of $n$ surfaces in $\mathbb{R}^{3}$ is a family of pseudoplanes in general position if

(i) The surfaces of $\Lambda$ are graphs of total bivariate continuous functions.

(ii) The intersection of any pair of surfaces in $\Lambda$ is a connected $x$-monotone unbounded curve.

(iii) Any triple of surfaces in $\Lambda$ intersect in exactly one point.

(iv) The $x y$-projections of the set of all $\left(\begin{array}{c}n \\ 2\end{array}\right)$ intersection curves of the surfaces form a family of pseudolines in the plane. That is, this is a collection of $\left(\begin{array}{l}n \\ 2\end{array}\right) x$-monotone unbounded curves, each pair of which intersect exactly once; see [3] for more details.

The assumption that the pseudoplanes of $\Lambda$ are in general position means that no point is incident to more than three pseudoplanes, no intersection curve of two pseudoplanes is tangent to a third pseudoplane, and no two pseudoplanes are tangent to each other. We note that conditions (i)-(iii) are natural, but condition (iv) might appear somewhat restrictive, although it obviously holds for planes. For any $a, b, c \in \Lambda$, we denote the intersection curve $a \cap b$ by $\gamma_{a, b}$, and the intersection point $a \cap b \cap c$ by $p_{a, b, c}$.

- Definition 3. Let $\gamma$ be a curve in $\mathbb{R}^{3}$. The vertical curtain through $\gamma$, denoted by $\Upsilon_{\gamma}$, is the collection of all z-vertical lines that intersect $\gamma$. The portion of $\Upsilon_{\gamma}$ above (resp., below) $\gamma$ is called the upper (resp., lower) curtain of $\gamma$, and is denoted by $\Upsilon_{\gamma}^{u}\left(\right.$ resp., $\left.\Upsilon_{\gamma}^{d}\right)$.

Let $\gamma$ be an $x$-monotone unbounded connected curve in $\mathbb{R}^{3}$, and let $p \in \gamma$. We call each of the two connected components of $\gamma \backslash\{p\}$ a half-curve of $\gamma$ emanating from $p$.

The following lemma is derived from the general position of the pseudoplanes in $\Lambda$ :

- Lemma 4. Let $a, b, c \in \Lambda$, and let $\gamma_{a, b}=a \cap b, p_{a, b, c}=a \cap b \cap c$.

(a) One of the two half-curves of $\gamma_{a, b}$ that emanates from $p_{a, b, c}$ lies fully below $c$, and the other half-curve lies fully above $c$.

(b) The collection of intersections between the surfaces of $\Lambda$ and $\Upsilon_{\gamma_{a, b}}$ forms an arrangement of unbounded $x$-monotone curves on $\Upsilon_{\gamma_{a, b}}$, each pair of which intersect at most once. ${ }^{2}$

Proof. The proof of (a) is straightforward and is omitted here. For (b), property (iv) implies that, for any $c, d \in \Lambda \backslash\{a, b\}$, the projection on the $x y$-plane of $\gamma_{a, b}$ and $\gamma_{c, d}=c \cap d$ intersect at most once. Thus, the intersection curves $c \cap \Upsilon_{\gamma_{a, b}}, d \cap \Upsilon_{\gamma_{a, b}}$ intersect at most once.

${ }^{2}$ In a sense, this is a collection of pseudolines, except that they are, in general, not drawn in a plane. 
Another property of $\mathcal{A}(\Lambda)$, shown in Agarwal and Sharir [2], is:

- Lemma 5. The complexity of the lower envelope of $\Lambda$ is $O(n)$.

The notion of corridors can easily be extended to the case of pseudoplanes. That is, for any $a, b, c \in \Lambda$, denote by $C_{a, b, c}$ the open region between the lower envelope and the upper envelope of $a, b, c$, and call it the corridor of $a, b, c$. Refer to corridors $C_{a, b, c}$ for which the intersection point $p_{a, b, c}$ lies at level $k$ as $k$-corridors, and define $C^{k}$ as the collection of $k$-corridors in $\mathcal{A}(\Lambda)$. The following is an extension of Definition 2:

- Definition 6. A corridor $C_{1}$ is immersed in a corridor $C_{2}$ if they share exactly one pseudoplane, and the intersection curve of the other two pseudoplanes of $C_{1}$ is fully contained in $C_{2}$. Let $X^{k}$ denote the number of ordered pairs of immersed corridors in $C^{k}$.

Organization of this section. In Section 2.1 we derive an upper bound for $X^{k}$, using an extended dual version of the Lovász Lemma. In Section 2.2 we obtain a lower bound for $X^{k}$, using a dual version of the Crossing Lemma. In Section 2.3 we combine those two bounds to obtain an upper bound on the complexity of the $k$-level of the arrangement.

\subsection{An extension of the dual version of the Lovász Lemma}

The following lemma is an extension to the case of pseudoplanes of a dual version of the antipodality property in the primal setup.

- Lemma 7. Let $\Lambda$ be as above, let $a, b \in \Lambda$, and let $\gamma_{a, b}=a \cap b$ denote their intersection curve. Let $h$ be a z-vertical line (i.e., parallel to the z-axis) that intersects $\gamma_{a, b}$ at some point p. Let $D=\Lambda \backslash\{a, b\}$, and $D^{k}=\left\{d \in D \mid C_{a, b, d} \in C^{k}\right\}$. Denote $h_{u p}=\left\{d \in D \mid z_{d \cap h}>z_{p}\right\}$ and $h_{\text {down }}=\left\{d \in D \mid z_{d \cap h}<z_{p}\right\}$ (by choosing p generically, we may assume that all these inequalities are indeed sharp). We then have ||$h_{u p} \cap D^{k}|-| h_{\text {down }} \cap D^{k}|| \leq 2$.

Proof. Denote the two half-curves of $\gamma_{a, b}$ emanating from $p$ by $\eta_{1}$ and $\eta_{2}$, and denote $D_{\eta_{1}}=\left\{d \in D \mid d\right.$ intersects $\left.\eta_{1}\right\}, D_{\eta_{2}}=\left\{d \in D \mid d\right.$ intersects $\left.\eta_{2}\right\}$. Clearly, with a generic choice of $p, D_{\eta_{1}} \cup D_{\eta_{2}}=D$, and since each triple of pseudoplanes in $\Lambda$ intersects only once, $D_{\eta_{1}} \cap D_{\eta_{2}}=\emptyset$. Enumerate the pseudoplanes in $D_{\eta_{1}}$ as $d_{1}, \ldots, d_{j}$, according to the order in which their respective intersection points with $\eta_{1}$, denoted $p_{1}, \ldots, p_{j}$, appear on $\eta_{1}$ in the direction from $p$ to the end of the half-curve. Assume there are $1 \leq r<s \leq j$ such that $d_{r}, d_{s} \in D_{\eta_{1}} \cap\left(h_{\text {down }} \cap D^{k}\right)$, and denote $D_{r, s}=\left\{d_{i} \in D_{\eta_{1}} \mid r \leq i \leq s\right\}$.

It is easy to show that, for each point $p_{i}$, the following properties hold (as they do in the case of planes. See the full version [17]); see Figure 3.

(i) $\lambda\left(p_{i}\right)=\lambda\left(p_{i-1}\right)-1$ if and only if both $d_{i-1}, d_{i} \in h_{\text {down }}$.

(ii) $\lambda\left(p_{i}\right)=\lambda\left(p_{i-1}\right)+1$ if and only if both $d_{i-1}, d_{i} \in h_{u p}$.

(iii) $\lambda\left(p_{i}\right)=\lambda\left(p_{i-1}\right)$ if and only if one of $d_{i-1}, d_{i}$ is in $h_{u p}$ and the other one is in $h_{\text {down }}$.

We claim that there must exist a pseudoplane $d^{\prime} \in D_{r, s}$ that is in $h_{u p} \cap D^{k}$. If $d_{r+1} \in h_{u p}$ then, by property (iii), $\lambda\left(d_{r+1}\right)=\lambda\left(d_{r}\right)=k$ and we are done. Otherwise, $d_{r+1} \in h_{\text {down }}$, and by (i) above, the level of $p_{r+1}$ is $k-1$. Note that $r+1<s$ because the level of $p_{s}$ is $k$. Because the level can change only by $0,+1$, or -1 between two consecutive points $p_{i}$, $p_{i+1}$, there must be a point $p_{i} \in D_{r, s} \subseteq D_{\eta_{1}}$, so that the level of $p_{i}$ is $k$ and the level of the previous point on $l_{a, b}$ is $k-1$, which means, by (ii) above, that $d_{i} \in h_{u p}$. That is, between each pair $p_{r}, p_{s} \in \eta_{1}$ so that $d_{r}, d_{s} \in h_{\text {down }} \cap D^{k}$, there exists $p_{i}$ so that $d_{i} \in h_{u p} \cap D^{k}$, and our claim is established. 

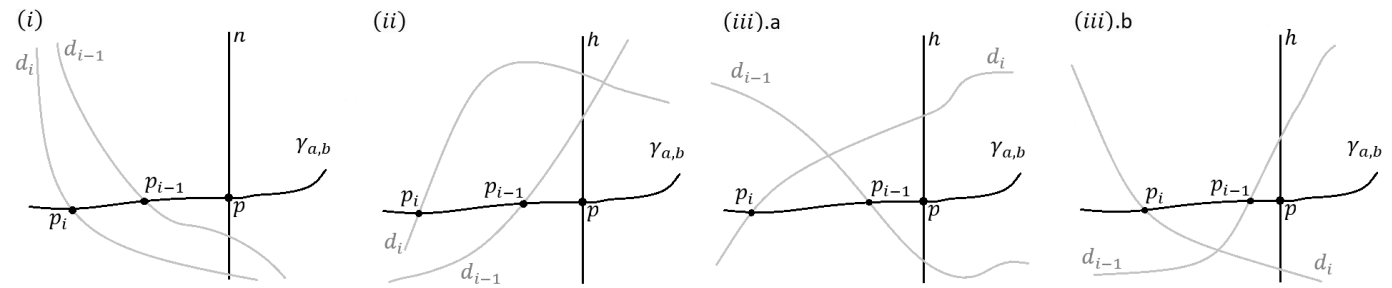

Figure 3 Both $d_{i}, d_{i-1}$ intersect the same half-curve of $\gamma_{a, b}$ emanating from $p$ : (i) The case where both $d_{i}, d_{i-1} \in h_{\text {down. }}$. (ii) The case where both $d_{i}, d_{i-1} \in h_{u p}$. (iii.a-b) The case where one of $d_{i}, d_{i-1}$ is in $h_{\text {down }}$, and the other one is in $h_{u p}$.

Similarly, between each pair $p_{r}, p_{s} \in \eta_{1}$ so that $d_{r}, d_{s} \in h_{u p} \cap D^{k}$, there exists $p_{i}$, for some $r<i<s$, so that $d_{i} \in h_{\text {down }} \cap D^{k}$. Both of these properties are easily seen to imply that

$$
|| h_{\text {up }} \cap D^{k} \cap D_{\eta_{1}}|-| h_{\text {down }} \cap D^{k} \cap D_{\eta_{1}}|| \leq 1 .
$$

The same reasoning applies to $\eta_{2}$, and yields ||$h_{u p} \cap D^{k} \cap D_{\eta_{2}}|-| h_{\text {down }} \cap D^{k} \cap D_{\eta_{2}}|| \leq 1$. Thus, ||$h_{u p} \cap D^{k}|-| h_{\text {down }} \cap D^{k}|| \leq 2$.

We next apply this lemma to obtain an extended dual version of the Lovász Lemma. Concretely, we derive an upper bound on the number of $k$-corridors that fully contain an intersection curve $\gamma_{a, b}=a \cap b$. In the case of planes (see [17]), the curve in question is the intersection line of two dual planes, which is dual to the line connecting the two corresponding primal points. The $k$-corridors are dual to the $k$-triangles in the primal, and a $k$-corridor fully contains $a \cap b$ if and only if the corresponding primal line crosses the corresponding primal $k$-triangle. The Lovász Lemma, in the primal, asserts that a line crosses at most $O\left(n^{2}\right)$ $k$-triangles. In the standard proof of the lemma, we translate a line from infinity towards the target line, and keep track of the number of $k$-triangles crossed by the line. This number changes only when the moving line sweeps through a segment connecting two input points, and we use the antipodality property to argue that the change in the number of crossed $k$-triangles at such an event is only \pm 1 , from which the lemma follows. In the dual setup, antipodality is replaced by a suitable version of Lemma 7 (for planes), and the sweeping of the primal line becomes a sweeping of the dual line, moving in a vertical plane from, say $+\infty$ towards the line $a \cap b$. The critical primal events are transformed into events where the moving line touches some intersection line $c \cap d$, for $c, d \in \Lambda \backslash\{a, b\}$. A suitable application of Lemma 7 then implies that the number of $k$-corridors that fully contain the moving curve changes by at most $\pm 2 .^{3}$

In the case of pseudoplanes, the sweeping is performed in the reverse order, from $\gamma_{a, b}$ upwards to a curve at $z=+\infty$. More importantly, the sweeping is no longer by translating (a copy of) $\gamma_{a, b}$, but follows the topological sweeping paradigm of Edelsbrunner and Guibas [11] (see also [12]); the sweep curve is always fully contained in the curtain $\Upsilon_{\gamma_{a, b}}$.

In the context considered here, we have an arrangement of curves within $\Upsilon_{\gamma_{a, b}}$, so that each pair of them intersects once, and sweep it with a curve $\gamma$, so that initially, and at every instance during the sweep, $\gamma$ intersects every other curve at most once. The sweep is a

3 The reason why in the dual the change is \pm 2 instead of \pm 1 in the primal is that, for convenience in the presentation, we allow the point $p$ in Lemma 7 to be somewhere at the middle of the curve; placing $p$ at the "end" of the curve (at $\pm \infty$ ) would make the change go down to \pm 1 . 
continuous motion of $\gamma$, given as a function $\tau \rightarrow \gamma_{\tau}$, for $\tau \in \mathbb{R}^{+}$, where $\gamma_{0}=\gamma$ is the initial placement of the sweeping curve and $\gamma_{\tau}$ approaches the curve $z=+\infty$ on $\Upsilon_{\gamma_{a, b}}$ as $\tau$ tends to $\infty$. Moreover, $\gamma_{\tau}$ lies fully below $\gamma_{\tau^{\prime}}$, for $\tau<\tau^{\prime}$.

The sweeping curve $\gamma$ is given a left-to-right orientation. Then $\gamma$ intersects some subset of the other curves of $\Gamma$ in some order. This ordered sequence changes when $\gamma$ passes through a vertex of the arrangement or when the set of curves intersecting $\gamma$ changes by an insertion or deletion of a curve, necessarily at the first or the last place in the sequence. We disregard the continuous nature of the sweep, and discretize it into a sequence of discrete steps, where each step represents one of these changes. As shown in [12], we have:

- Lemma 8 (Hershberger and Snoeyink [12]). Any planar arrangement of a set $\Gamma$ of bi-infinite curves, any pair of which intersect at most once, can be swept topologically, starting with any curve $\gamma \in \Gamma$, so that, at any time during the sweep, the sweeping curve intersects any other curve at most once.

Although in our case the sweep takes place within $\Upsilon_{\gamma_{a, b}}$, which is not a plane in general, we can flatten it in the $x$-direction into a plane, keeping the $z$-vertical direction unchanged, and thereby apply the topological sweeping machinery of $[11,12]$ within this curtain.

The sweeping mechanism, as described in [12], proceeds in a sequence of discrete steps, each of which implements one of three kinds of local moves, listed below, allowing to advance the sweeping curve past an intersection point, and to add or to remove curves from the set of curves intersected by the sweeping curve, without violating the 1 -intersection property.

Let $c \in \Gamma$ be the sweeping curve, and denote by $\Xi(c)=\left(c_{1}, c_{2}, c_{3}, \ldots\right)$ the sequence of curves of $\Gamma$ that intersect $c$, sorted in the left-to-right order of their intersections along $c$. The basic steps of the sweep are of the following three types (see Figure 4):

(i)

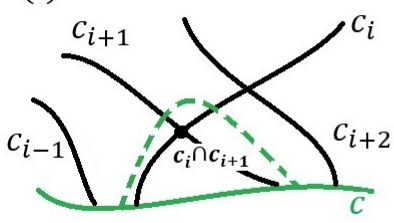

(ii)

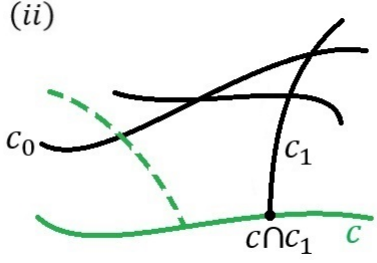

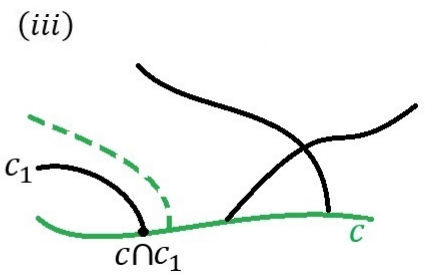

Figure 4 Operations by which the sweep progresses: Taking on the first ray. (iii) Passing over the first ray.

1. Passing over an empty triangle: We have a consecutive pair of curves $c_{i}, c_{i+1}$ along $c$ that intersect above $c$, and no other curve passes through the triangle formed by $c, c_{i}, c_{i+1}$. Then $c$ can move past the intersection point of $c_{i}, c_{i+1}$. See Figure 4(i).

2. Taking on the first ray: We have a curve $c_{0}$ that does not intersect $c$, but $c_{0}$ and $c$ are adjacent on the left (i.e., at $x=-\infty$ ). Then we can move $c$ upwards, make it intersect $c_{0}$ at a point that lies to the left of all other intersection points, both on $c$ and on $c_{0}$. This increases the intersection sequence $\Xi(c)$ by one element, now its first element.

3. Passing over the first ray: Here the first intersection point along $c$ is with a curve $c_{1}$ so that $c \cap c_{1}$ is also the first intersection along $c_{1}$ from the left. Then $c$ can move upwards, disentangling itself from $c_{1}$, and losing its intersection point with $c_{1}$, this time removing his first element of $\Xi(c)$.

As shown in [12], we can implement the sweep so that it only performs steps of these three types, and does not have to perform the symmetric operations to (ii) and (iii), of taking on or passing over the last ray. 
We now establish the generalized dual version of the Lovász Lemma.

- Lemma 9. Any bi-infinite $x$-monotone curve $\gamma_{0}$ such that (i) $\gamma_{0}$ intersects each pseudoplane in $\Lambda$ in exactly one point, and (ii) the xy-projection of $\gamma_{0}$ intersects the xy-projection of any intersection curve of two surfaces of $\Lambda$ at most once, is fully contained in at most $n(n-1) / 2$ corridors in $C^{k}$.

Proof. Let $\gamma_{0}$ be a curve as in the lemma, and consider the vertical curtain $\Upsilon_{\gamma_{0}}$ that it spans. For each pseudoplane $a \in \Lambda$, denote by $\sigma_{a}$ the intersection curve $a \cap \Upsilon_{\gamma_{0}}$, and put $\Sigma=\left\{\sigma_{a} \mid a \in \Lambda\right\}$. By the assumptions of the lemma, the collection of bi-infinite curves $\left\{\gamma_{0}\right\} \cup \Sigma$ has the property that any two curves in this family intersect at most once. Moreover, as already remarked earlier, by regarding the $x y$-projection $\gamma_{0}^{*}$ of $\gamma_{0}$ as a homeomorphic copy of the real line, we can identify $\Upsilon_{\gamma_{0}}$ as a homeomorphic copy of a vertical plane, where vertical lines are mapped to vertical lines. It follows that we can apply Lemma 8 to the arrangement of $\left\{\sigma_{a} \mid a \in \Lambda\right\}$ within $\Upsilon_{\gamma_{0}}$, and conclude that this arrangement can be topologically swept with a curve that starts at $\gamma_{0}$ and proceeds upwards, to infinity.

Denote by $\gamma_{\tau}$ the sweeping curve at some moment $\tau$, where the curve coincides with $\gamma_{0}$ at $\tau=0$. At the beginning of the sweep, $\gamma_{0}$ is fully contained in some number $Y$ of $k$-corridors, and at the end of the sweep, $\gamma_{\tau}$ is not contained in any of the corridors in $C^{k}$. We will establish an upper bound on the difference between the number of $k$-corridors that $\gamma_{\tau}$ gets out of (i.e., stops being fully contained in) and the number of $k$-corridors that it gets into (i.e., starts being fully contained in), at any critical event during the sweep. Summing those differences will yield the asserted upper bound on $Y$.

Consider $\gamma_{\tau}$ at some instance $\tau$ during the sweep, and let $a \in \Lambda$. If $\sigma_{a}$ is fully above $\gamma_{\tau}$, we get that $\gamma_{0}$, which is obtained by some motion of $\gamma_{\tau}$ downwards, is fully below $a$, a contradiction to the assumption that $\gamma_{0}$ intersects all the pseudoplanes in $\Lambda$. Therefore, each pseudoplane in $\Lambda$ is either fully below $\gamma_{\tau}$, or intersects it (exactly once). Hence, during the sweeping from $\gamma_{0}$, the only valid sweeping steps are passing over an empty triangle and passing over the first ray.

Clearly, it suffices to consider what happens at instances $\tau$ at which $\gamma$ is about to pass through a vertex of the arrangement of $\left\{\sigma_{a} \mid a \in \Lambda\right\}$ on $\Upsilon_{\gamma_{0}}$, or at instances at which $\gamma_{\tau}$ is about to pass over the first ray. So let $\tau^{-}$and $\tau^{+}$denote instances immediately before and after such a critical transition. We distinguish between three types of sweeping steps.
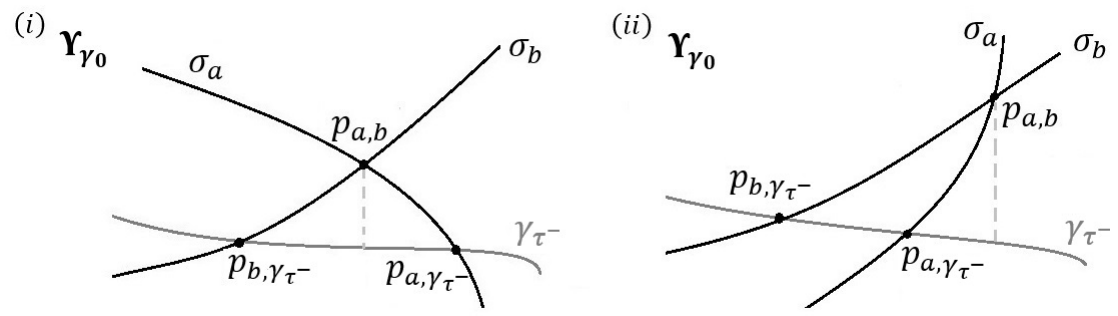

Figure 5 (i) The intersection point $p_{a, b}=\sigma_{a} \cap \sigma_{b}$ is directly above the curve $\gamma_{\tau^{-}}$somewhere between $p_{a, \gamma_{\tau^{-}}}=\sigma_{a} \cap \gamma_{\tau^{-}}$and $p_{b, \gamma_{\tau^{-}}}=\sigma_{b} \cap \gamma_{\tau^{-}}$. (ii) The intersection point $p_{a, b}$ is not directly above any point on the curve $\gamma_{\tau^{-}}$between $p_{a, \gamma_{\tau^{-}}}$and $p_{b, \gamma_{\tau^{-}}}$.

Case 1: The transition at $\tau$ is that we pass over an empty triangle, defined by some pair of curves $\sigma_{a}, \sigma_{b}$ and $\gamma_{\tau^{-}}$, such that the point on $\gamma_{\tau^{-}}$directly below the intersection point $p_{a, b}$ is somewhere between $p_{a, \gamma_{\tau^{-}}}$and $p_{b, \gamma_{\tau^{-}}}$(see Figure 5(i)). Since $\gamma_{\tau}$ intersects each of 
$\sigma_{a}, \sigma_{b}$ at most once, almost all of the curve $\gamma_{\tau^{-}}$is between the lower envelope and the upper envelope of $\left\{\sigma_{a}, \sigma_{b}\right\}$, except for its portion between $p_{a, \gamma_{\tau^{-}}}$and $p_{b, \gamma_{\tau^{-}}}$. Since the triangle defined by $\sigma_{a}, \sigma_{b}$ and $\gamma_{\tau^{-}}$is empty, each curve in $\Sigma$ that lies below $p_{a, b}$, defines a corridor with $\sigma_{a}, \sigma_{b}$, such that $\gamma_{\tau^{-}}$lies fully in that corridor. Symmetrically, $\gamma_{\tau^{+}}$, for $\tau^{+}$ sufficiently close to $\tau$, lies fully in each corridor defined by $\sigma_{a}, \sigma_{b}$ and a curve in $\Sigma$ that passes above $p_{a, b}$ (see, e.g., Figure 6(i)). Hence, by Lemma 7, the absolute value of the difference between the number of $k$-corridors that $\gamma_{\tau}$ gets out of at $\tau$, and the number of $k$-corridors that $\gamma_{\tau}$ gets into, is at most 2 .
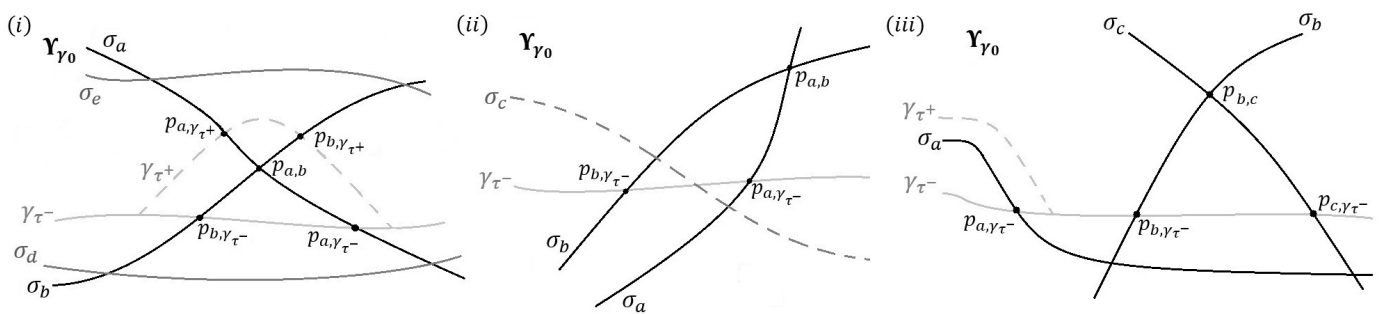

Figure 6 The three cases of critical events during the sweep.

Case 2: The transition at $\tau$ is that we pass over an empty triangle, defined by $\sigma_{a}, \sigma_{b}$ and $\gamma_{\tau^{-}}$, where now the vertical projection of the intersection point $p_{a, b}$ onto $\gamma_{\tau^{-}}$is not between $p_{a, \gamma_{\tau^{-}}}$and $p_{b, \gamma_{\tau^{-}}}$, but lies on one side of both, say past $p_{a, \gamma_{\tau^{-}}}$to the right (see Figure $5(\mathrm{ii}))$. We claim that neither $\gamma_{\tau^{-}}$nor $\gamma_{\tau^{+}}$is fully contained in any corridor $C_{a, b, c}$, for $\sigma_{c} \in \Sigma$. Indeed (refer again to Figure 5 ), by Lemma 4, the half-curve emanating from $p_{a, \gamma_{\tau^{-}}}$on $\gamma_{\tau^{-}}$to the right is below the lower envelope of $\left\{\sigma_{a}, \sigma_{b}\right\}$, and the half-curve emanating from $p_{b, \gamma_{\tau^{-}}}$on $\gamma_{\tau^{-}}$to the left is above the upper envelope of $\left\{\sigma_{a}, \sigma_{b}\right\}$. Hence, in order for $\gamma_{\tau^{-}}$to be fully contained in a corridor $C_{a, b, c}$ for some $\sigma_{c} \in \Sigma, \sigma_{c}$ must pass above $p_{b, \gamma_{\tau^{-}}}$and below $a_{b, \gamma_{\tau^{-}}}$, and therefore it must intersect the triangle defined by $\sigma_{a}, \sigma_{b}$ and $\gamma_{\tau^{-}}$(see Figure $6($ ii $)$ ). Since this triangle is empty, there is no such $\sigma_{c}$. Symmetrically ${ }^{4}, \gamma_{\tau^{+}}$is not contained in any corridor $C_{a, b, c}$ for any $c$. Hence, at this step in the sweeping process, there is no change in the set of corridors that fully contain $\gamma_{\tau}$.

Case 3: The transition at $\tau$ is passing over the first ray, belonging to some $\sigma_{a} \in \Sigma$. We claim that here too $\gamma_{\tau^{-}}$and $\gamma_{\tau^{+}}$are fully contained in the same corridors. Indeed, except for the left ray of $\sigma_{a}, \gamma_{\tau^{-}}$and $\gamma_{\tau^{+}}$are fully above $\sigma_{a}$. Moreover, the only corridors that $\gamma$ can get into or out of at this transition must involve $\sigma_{a}$. Let $C_{a, b, c}$ be such a corridor. If $\sigma_{a}$ appears on both the upper and the lower envelopes of $\left\{\sigma_{a}, \sigma_{b}, \sigma_{c}\right\}$ then, as is easily checked, neither $\gamma_{\tau^{-}}$nor $\gamma_{\tau^{+}}$can be fully contained in $C_{a, b, c}$. Hence, $\sigma_{a}$ must appear on exactly one of the envelopes, and then it must appear there as the middle portion of the envelope (see Figure 6(iii)). But then the left ray of $\sigma_{a}$ over which $\gamma$ is swept cannot appear on either envelope, so the transition does not cause $\gamma$ to enter or leave $C_{a, b, c}$, as claimed.

In summary, the only kind of step during the sweep in which the number of $k$-corridors that the sweeping curve is contained in changes, is passing over an empty triangle with the structure considered in Case 1 and then, as argued above, this number can change by at most 2. There are $\left(\begin{array}{l}n \\ 2\end{array}\right)=\frac{n(n-1)}{2}$ intersection points on $\Upsilon_{\gamma_{0}}$, since each pair of curves in $\Sigma$

\footnotetext{
${ }^{4}$ Indeed, right after the transition, $\sigma_{a}, \sigma_{b}$ and $\gamma_{\tau^{+}}$form an empty triangle above $p_{a, b}$, with similar properties that allow us to apply a symmetric variant of the argument just presented.
} 
intersects at most once. Assume without loss of generality that at most half of them are above $\gamma_{0}$ (otherwise, consider sweeping $\gamma_{0}$ in the opposite direction, downwards to infinity). The sweeping curve can encounter at most $\frac{1}{2} \cdot \frac{n(n-1)}{2}$ empty triangles whose middle vertex lies above the opposite edge. Thus, at the beginning of the process, $\gamma_{0}$ is fully contained in at most $\frac{n(n-1)}{2} k$-corridors and the claim follows.

As a corollary (see also the full version [17]), we obtain the following upper bound on $X^{k}$ :

- Lemma 10. The number $X^{k}$ of ordered pairs of $k$-corridors such that the first corridor is immersed in the second one, in the arrangement $\mathcal{A}(\Lambda)$, is at most $\frac{3 n^{4}}{4}$.

Proof. Fix an intersection curve $\gamma_{a, b}=a \cap b$ of two pseudoplanes from $\Lambda$. By Lemma $9, \gamma_{a, b}$ is fully contained in at most $n(n-1) / 2 k$-corridors. For each containing $k$-corridor $C_{c, d, e}, \gamma_{a, b}$ can contribute at most three ordered pairs to $X^{k}$, namely an immersion of $C_{a, b, c}$ in $C_{c, d, e}$, of $C_{a, b, d}$ in $C_{c, d, e}$, and of $C_{a, b, e}$ in $C_{c, d, e}$. Since there are only $\left(\begin{array}{l}n \\ 2\end{array}\right)$ intersection curves in $\mathcal{A}(\Lambda)$, we get that there are at most $3 \frac{n(n-1)}{2}\left(\begin{array}{l}n \\ 2\end{array}\right)<\frac{3 n^{4}}{4}$ ordered pairs of immersed $k$-corridors.

\subsection{The dual version of the Crossing Lemma}

In this subsection we derive a lower bound on $X^{k}$, using a dual version of the Crossing Lemma (see [4]), extended to the case of pseudoplanes. For each pseudoplane $a \in \Lambda$, denote by $z_{a}$ the intersection point of $a$ with the $z$-axis. We can choose the position of the $z$-axis so as to ensure that (a) all the values $z_{a}$ are distinct and (b) for each $a \in \Lambda, z_{a}$ lies above (in the $y$-direction of the $x y$-projection of $a$ ) all the intersection curves $\gamma_{a, b}$, for $b \in \Lambda \backslash\{a\}$.

- Definition 11. Let $a \in \Lambda$. Denote by $\Gamma_{a}$ the collection of the intersection curves of a and the other pseudoplanes $b \in \Lambda$ with $z_{b}>z_{a}$. That is, $\Gamma_{a}=\left\{\gamma_{b}:=a \cap b \mid b \in \Lambda \backslash\{a\}, z_{b}>z_{a}\right\}$.

By the assumptions on $\Lambda$, the $x y$-projection of any intersection curve of two pseudoplanes in $\Lambda$ is an $x$-monotone curve. Therefore, $\Gamma_{a}$ forms a family of $x$-monotone curves on the surface $a$. Since $a$ is the graph of a bivariate continuous function, it will be convenient to identify it with its $x y$-projection, and think of it, for the purpose of the current analysis, as a horizontal plane. Each pair of curves from $\Gamma_{a}$ intersects exactly once, because each triple of pseudoplanes in $\Lambda$ intersects exactly once. Each curve in $\Gamma_{a}$ is bi-infinite and divides $a$ into two unbounded regions. These considerations allow us to interpret $\Gamma_{a}$ as a family of $x$-monotone pseudolines in the plane.

- Definition 12. Let $a \in \Lambda$, and let $\Gamma_{a}$ be as above. Each $d \in \Lambda \backslash\{a\}$ for which $\gamma_{d} \in \Gamma_{a}$ divides a into two disjoint regions: the region $a_{d}^{-}$on a that is fully above the pseudoplane $d$, and the region $a_{d}^{+}$on a that is fully below $d$ (so $a_{d}^{-}$means that $d$ is below $a$, and $a_{d}^{+}$means that $d$ is above a). These two regions are delimited by the intersecion curve $\gamma_{d}$ on a. Note that $z_{a} \in a_{d}^{+}$. That is, $a_{d}^{+}$is the region that lies above (in the $y$-direction) the intersection curve $\gamma_{d}$, and $a_{d}^{-}$is the region below $\gamma_{d}$.

For each pair of distinct pseudoplanes $b, c \in \Lambda \backslash\{a\}$ such that $\gamma_{b}, \gamma_{c} \in \Gamma_{a}$, define the $\mathbf{x}$-horizontal wedge $\boldsymbol{W}_{b, c}^{a}$ as the region on the pseudoplane a that is contained in exactly one of the two regions $a_{b}^{+}, a_{c}^{+}$, that is, in exactly one of the regions that are bounded by $\gamma_{b}, \gamma_{c}$ and contain $z_{a}$ (see Figure 7(1)).

Note that our assumption on the position of $z_{a}$ in $a$ allows to regard the wedges $W_{b, c}^{a}$ as being indeed " $x$-horizontal" within the $x y$-frame in $a$. 
Continue to fix the pseudoplane $a$, let $E_{a}$ be some subset of vertices of $\mathcal{A}\left(\Gamma_{a}\right)$, and let $G_{a}=\left(\Gamma_{a}, E_{a}\right)$ denote the graph whose vertices are the pseudolines in $\Gamma_{a}$ and whose edges are the pairs that form the vertices of $E_{a}$. A diamond in $G_{a}$ is two pairs $\left\{\gamma_{b}, \gamma_{c}\right\},\left\{\gamma_{d}, \gamma_{e}\right\}$ of curves of $\Gamma_{a}$ on $a$, both pairs belonging to $E_{a}$, with all four pseudoplanes $b, c, d$, e distinct, such that $p_{a, b, c}=\gamma_{b} \cap \gamma_{c} \in W_{d, e}^{a}$ and $p_{a, d, e}=\gamma_{d} \cap \gamma_{e} \in W_{b, c}^{a}$. See Figure $7(2 . \mathrm{i})$ and $7(2 . \mathrm{ii})$.

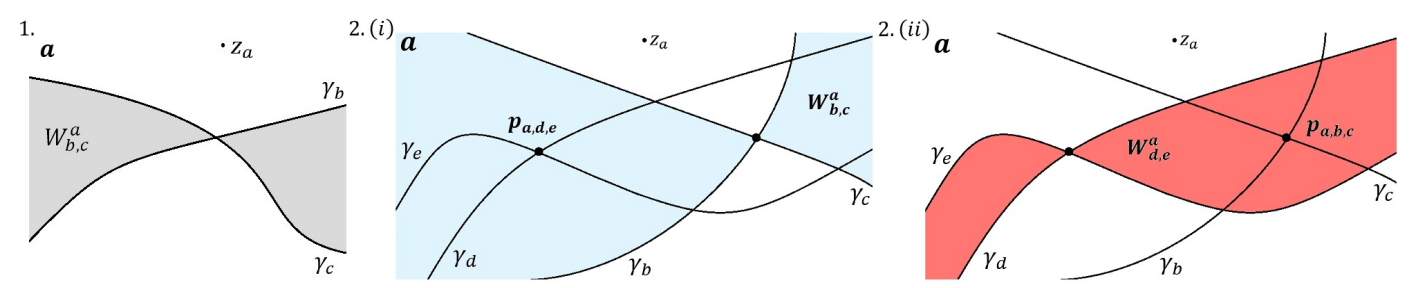

Figure 71 . The $x$-horizontal wedge $W_{b, c}^{a}$ on the pseudoplane $a$. The regions $a_{b}^{+}$and $a_{c}^{+}$lie above (in the $y$-direction) the respective curves $\gamma_{b}$ and $\gamma_{c}$, and they both contain $z_{a}$. 2. The two pairs $\left\{\gamma_{b}, \gamma_{c}\right\},\left\{\gamma_{d}, \gamma_{e}\right\}$ on $a$ form a diamond in $G_{a}$. (i) The blue area is the $x$-horizontal wedge $W_{b, c}^{a}$, and it contains $p_{a, d, e}=\gamma_{d} \cap \gamma_{e}$. (ii) The red area is the $x$-horizontal wedge $W_{d, e}^{a}$, and it contains $p_{a, b, c}=\gamma_{b} \cap \gamma_{c}$.

The following is our version of an extension of the dual version of Euler's formula for planar maps, derived in Tamaki and Tokuyama [18], for the case of pseudolines in $\mathbb{R}^{2}$ :

- Lemma 13 (Tamaki and Tokuyama [18]). For a pseudoplane $a \in \Lambda$, let $G_{a}$ be as defined above, with $\left|\Gamma_{a}\right|>3$. If $\Gamma_{a}$ is diamond-free, then $G_{a}$ is planar, and so $\left|E_{a}\right| \leq 3\left|\Gamma_{a}\right|-6$.

As a corollary of Lemma 13, we have:

- Lemma 14 (Generalized dual version of the Crossing Lemma). Let $\Gamma_{a}$ and $G_{a}$ be as above, so that $\left|E_{a}\right|>4\left|\Gamma_{a}\right|$. The number of diamonds in $G_{a}$ is at least $\frac{\left|E_{a}\right|^{3}}{64\left|\Gamma_{a}\right|^{2}}$.

The proof follows more or less the standard probabilistic proof of the Crossing Lemma. That is, Lemma 13 easily implies that the number of diamonds in $G_{a}$ is at least $\left|E_{a}\right|-$ $3\left|\Gamma_{a}\right|+6>\left|E_{a}\right|-3\left|\Gamma_{a}\right|$. One then draws a random sample $G_{a}^{\prime}$ of $G_{a}$, by picking each curve $\gamma_{b} \in \Gamma_{a}$ independently with probability $p=4\left|\Gamma_{a}\right| /\left|E_{a}\right|$, and applies the above bootstrapping bound to $G_{a}^{\prime}$, to obtain the improved bound in the lemma. This works, as in the planar case, provided that $\left|E_{a}\right|>4\left|\Gamma_{a}\right|$, as the lemma assumes.

We now specialize this result to our context. For each $a \in \Lambda$, consider the set $E_{a}^{k}=$ $\left\{p_{a, b, c}=\gamma_{b} \cap \gamma_{c} \mid \gamma_{b}, \gamma_{c} \in \Gamma_{a}, C_{a, b, c} \in C^{k}\right\}$, and the graph $G_{a}^{k}=\left(\Gamma_{a}, E_{a}^{k}\right)$ defined as above. Lemma 14 implies the following:

- Lemma 15. The number $X^{k}$ of ordered pairs of immersed $k$-corridors in the arrangement $\mathcal{A}(\Lambda)$ is at least $\frac{\left|C^{k}\right|^{3}}{64 n^{4}}-n^{2}$.

Proof. Let $a \in \Lambda, G_{a}^{k}=\left(\Gamma_{a}, E_{a}^{k}\right)$ be as above, and define $\Delta_{a}$ as the number of diamonds in $G_{a}^{k}$. Let $\left\{\gamma_{b}, \gamma_{c}\right\},\left\{\gamma_{d}, \gamma_{e}\right\}$ be a pair that form a diamond. Since the pseudoplanes in $\Lambda$ satisfy property (iv) and are in general position, the $x y$-projections of the curves $\gamma_{b, c}$ and $\gamma_{d, e}$ have exactly one intersection point (but $\gamma_{b, c}$ and $\gamma_{d, e}$ do not intersect in 3-space). Moreover, since $b, c$ are the graphs of total bivariate functions, the projection of their intersection curve $\gamma_{b, c}$ on $a$ is fully contained in the region $\left\{p_{a, b, c}\right\} \cup\left\{a_{b}^{+} \cap a_{c}^{+}\right\} \cup\left\{a_{b}^{-} \cap a_{c}^{-}\right\}$. That is, the projection of $\gamma_{b, c}$ is disjoint from the interior of $W_{b, c}^{a}$. Similarly, the projection of the intersection curve $\gamma_{d, e}$ on $a$ is fully contained in the region $\left\{p_{a, d, e}\right\} \cup\left\{a_{d}^{+} \cap a_{e}^{+}\right\} \cup\left\{a_{d}^{-} \cap a_{e}^{-}\right\}$, and is disjoint from $W_{d, e}^{a}$. In addition, the portion of $\gamma_{b, c}$ that projects to $a_{b}^{+} \cap a_{c}^{+}$lies above $a$ and the portion projecting to $a_{b}^{-} \cap a_{c}^{-}$lies below $a$. A similar property holds for $\gamma_{d, e}$. 
Assume without loss of generality that the pseudoplanes $b, c, d, e$ intersect $a$ as in Figure 8(i); that is, $p_{a, b, c}$ is contained in $a_{d}^{-} \cap a_{e}^{+}$and $p_{a, d, e}$ is contained in $a_{b}^{-} \cap a_{c}^{+}$. Since $\left\{\gamma_{b}, \gamma_{c}\right\}$ and $\left\{\gamma_{d}, \gamma_{e}\right\}$ form a diamond and each pair of curves on $a$ intersects exactly once, the intersection of the boundary of $a_{d}^{+} \cap a_{e}^{+}$and the boundary of $a_{b}^{-} \cap a_{c}^{-}$is empty. Indeed, the interior of the arc $p_{a, b, c} p_{a, d, e}$ is fully contained in $a_{b}^{-} \cap a_{c}^{+}$, and the half-curve of $\gamma_{d}$ emanating from $p_{a, b, d}$ and not containing $p_{a, d, e}$, is fully contained in $a_{b}^{+}$(otherwise, $\gamma_{d}$ would intersect $\gamma_{b}$ more than once). On the other hand, the half-curve of $\gamma_{e}$ emanating from $p_{a, d, e}$ and not containing $p_{a, c, e}$, is fully contained in $a_{c}^{+}$, since $\gamma_{c}$ already intersects the other half-curve of $\gamma_{e}$. These two observations establish our claim. The regions $a_{b}^{-} \cap a_{c}^{-}$and $a_{d}^{+} \cap a_{e}^{+}$are not contained in one another, and therefore their intersection is empty. Similarly, The intersection of $a_{b}^{+} \cap a_{c}^{+}$ and $a_{d}^{-} \cap a_{e}^{-}$is empty.

(i)

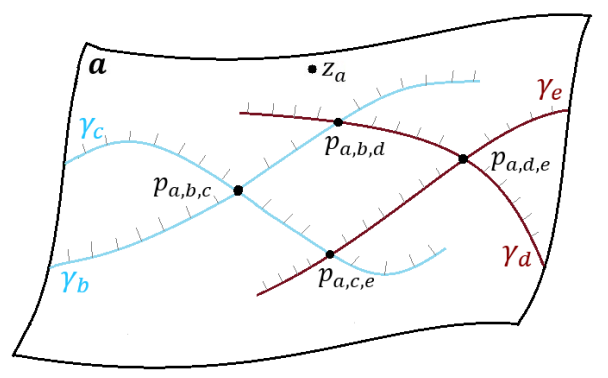

(ii)

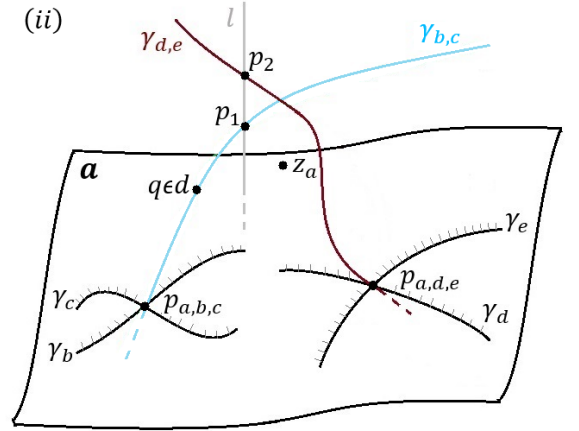

Figure 8 The two pairs $\left\{\gamma_{b}, \gamma_{c}\right\},\left\{\gamma_{d}, \gamma_{e}\right\}$ on $a$ form a diamond in $G_{a}^{k}$. (i) The diamond, where $p_{a, b, c}$ is contained in $a_{d}^{-} \cap a_{e}^{+}$and $p_{a, d, e}$ is contained in $a_{b}^{-} \cap a_{c}^{+}$. (ii) The intersection curve $\gamma_{b, c}$ is below the intersection curve $\gamma_{d, e}$. The pseudoplane $d$ (resp., e) meets $\gamma_{b, c}$ at a point $q$ between $p_{a, b, c}$ and $p_{1}$ (resp., at a point, not drawn, outside this arc).

Assume without loss of generality that $\gamma_{b, c}$ passes below $\gamma_{d, e}$. That is, letting $l$ denote the unique $z$-vertical line that meets both $\gamma_{b, c}, \gamma_{d, e}$, the points $p_{1}=l \cap \gamma_{b, c}, p_{2}=l \cap \gamma_{d, e}$ satisfy $z_{p_{1}}<z_{p_{2}}$. Assume without loss of generality that $l$ intersects $a$ in the region on $a$ that is the intersection of $a_{b}^{+}, a_{c}^{+}, a_{d}^{+}, a_{e}^{+}$(the regions on $a$ induced by the curves $\gamma_{b}, \gamma_{c}, \gamma_{d}, \gamma_{e}$ and containing $z_{a}$ ). The case where $l$ intersects $a$ in the region on $a$ that is the intersection of $a_{b}^{-}, a_{c}^{-}, a_{d}^{-}, a_{e}^{-}$, is handled symmetrically. These are the only two possibilities, since the intersection of $a_{b}^{+}, a_{c}^{+}, a_{d}^{-}, a_{e}^{-}$is empty, and so is the intersection of $a_{b}^{-}, a_{c}^{-}, a_{d}^{+}, a_{e}^{+}$.

Since $\gamma_{d, e}$ is above $p_{1}$, it follows that both $d$ and $e$ themselves are above $p_{1}$. Moreover, since $p_{a, b, c}$ lies in $a_{d}^{-}, d$ must lie below $p_{a, b, c}$. Hence $d$ must intersect $\gamma_{b, c}$ at some point $q$ between $p_{a, b, c}$ and $p_{1}$. Moreover, since $e$ satisfies $z_{e}>z_{a}$ and $p_{a, b, c}$ lies in $a_{e}^{+}$, as in Figure 8(ii), $e$ is above $p_{a, b, c}$. Since $e$ is also above $p_{1}$ and $e$ is the graph of a bivariate continuous function, its single intersection point with $\gamma_{b, c}$ must be outside the arc $p_{a, b, c} p_{1}$ of $\gamma_{b, c}$.

We claim that $\gamma_{b, c} \subseteq C_{a, d, e}$. Indeed, $\gamma_{b, c}$ is fully above the lower envelope of $\{a, d, e\}$ : the half-curve of $\gamma_{b, c}$ that emanates from $p_{a, b, c}$ and contains $p_{1}$ lies above the pseudoplane $a\left(\gamma_{b, c}\right.$ intersects $a$ at $\left.p_{a, b, c}\right)$, and the complementary half-curve lies above $d$, because the intersection point $q$ of $\gamma_{b, c}$ and $d$ lies between $p_{a, b, c}$ and $p_{1}$. The intersection curve $\gamma_{b, c}$ also lies fully below the upper envelope of $\{a, d, e\}$. That is because (i) the half-curve of $\gamma_{b, c}$ that emanates from the intersection point $q$ of $\gamma_{b, c}$ and $d$, and contains $p_{1}$, lies below the pseudoplane $d$, since $p_{2} \in d$ is higher than $p_{1}$; (ii) the half-curve of $\gamma_{b, c}$ that emanates from $p_{a, b, c}$ and does not contain $p_{1}$, lies below the pseudoplane $a$ (again, $\gamma_{b, c}$ intersects $a$ at $p_{a, b, c}$ ); and (iii) the arc $p_{a, b, c} q$ is below $e$, since $e$ is above both $p_{a, b, c}, p_{1}$ and therefore must be above the complete arc $p_{a, b, c} p_{1}$, and in particular $e$ is above the smaller arc $p_{a, b, c} q$. The other cases behave similarly and lead to similar conclusions. 
Thus for each pair $\left\{\gamma_{b}, \gamma_{c}\right\},\left\{\gamma_{d}, \gamma_{e}\right\}$ that form a diamond, either $\gamma_{b, c} \subseteq C_{a, d, e}$, or $\gamma_{d, e} \subseteq$ $C_{a, b, c}$. Either way, one of the corridors $C_{a, b, c}, C_{a, d, e}$ is immersed in the other one. Notice that every diamond in $\left\{G_{a}^{k}\right\}_{a \in \Lambda}$ yields a distinct ordered pair of immersed $k$-corridors, because for each $k$-corridor $C_{a, b, c}$, the intersection point $p_{a, b, c}=a \cap b \cap c$ represents an edge of only the graph associated with the pseudoplane with the lowest intersection point with the $z$-axis. Hence, by the dual version of the Crossing Lemma, namely Lemma 14, we have $X^{k} \geq \sum_{a} \frac{\left|E_{a}^{k}\right|^{3}}{64\left|\Gamma_{a}\right|^{2}}$, where the sum is over all those $a$ for which $\left|E_{a}^{k}\right| \geq 4\left|\Gamma_{a}\right|$. Any other pseudoplane $a$ satisfies $\frac{\left|E_{a}^{k}\right|^{3}}{64\left|\Gamma_{a}\right|^{2}} \leq\left|\Gamma_{a}\right|$, which implies the somewhat weaker lower bound

$$
X^{k} \geq \sum_{a \in \Lambda}\left(\frac{\left|E_{a}^{k}\right|^{3}}{64\left|\Gamma_{a}\right|^{2}}-\left|\Gamma_{a}\right|\right)
$$

By the definition of $G_{a}^{k}$, and as just noted, each $k$-corridor $C_{a, b, c}$ in $\mathcal{A}(\Lambda)$ appears in exactly one of $E_{a}^{k}, E_{b}^{k}, E_{c}^{k}$ (in the graph of the pseudoplane that intersects the $z$-axis at the lowest point among the three). Thus, $\sum_{a \in \Lambda}\left|E_{a}^{k}\right|=\left|C^{k}\right|$. The number of curves in $\Gamma_{a}$ is at most $n-1$. Therefore, using (1) and Hölder's inequality, we get the following lower bound $X^{k} \geq \sum_{a \in \Lambda}\left(\frac{\left|E_{a}^{k}\right|^{3}}{64\left|\Gamma_{a}\right|^{2}}-\left|\Gamma_{a}\right|\right) \geq \frac{1}{64 n^{2}} \sum_{a \in \Lambda}\left|E_{a}^{k}\right|^{3}-n^{2} \geq \frac{1}{64 n^{2}} \cdot \frac{\left(\sum_{a \in \Lambda}\left|E_{a}^{k}\right|\right)^{3}}{n^{2}}-n^{2}=\frac{\left|C^{k}\right|^{3}}{64 n^{4}}-n^{2}$.

\subsection{The complexity of the $k$-level of $\mathcal{A}(\Lambda)$}

We are now ready to obtain the upper bound on the complexity of the $k$-level of $\mathcal{A}(\Lambda)$.

- Lemma 16. The complexity of the k-level of $\mathcal{A}(\Lambda)$ is $O\left(n^{8 / 3}\right)$.

Proof. We compare the upper bound in Lemma 10 and the lower bound in Lemma 15 for the number $X^{k}$ of ordered pairs of immersed $k$-corridors in $\mathcal{A}(\Lambda)$, and get:

$$
\frac{3 n^{4}}{4} \geq X^{k} \geq \frac{\left|C^{k}\right|^{3}}{64 n^{4}}-n^{2} .
$$

Hence we get that $\left|C^{k}\right|^{3} \leq 48 n^{8}+64 n^{6}$, which implies that $\left|C^{k}\right|=O\left(n^{8 / 3}\right)$. The number of $k$-corridors is the number of vertices of $\mathcal{A}(\Lambda)$ at level $k$, which implies that the complexity of the $k$-level of $\mathcal{A}(\Lambda)$ is $O\left(n^{8 / 3}\right)$.

Combining the upper bound in Lemma 16 with the general technique of [1], we get the following $k$-sensitive result, whose proof is reviewed in the full version [17].

- Theorem 17. The complexity of the k-level of $\mathcal{A}(\Lambda)$ is $O\left(n k^{5 / 3}\right)$.

\section{Discussion}

In this paper we have shown that, for any set $\Lambda$ of $n$ surfaces in $\mathbb{R}^{3}$ that form a family of pseudoplanes, in the sense of satisfying properties (i)-(iv) of Section 2, the complexity of the $k$-level of $\mathcal{A}(\Lambda)$ is $O\left(n k^{5 / 3}\right)$. Our analysis is based on ingredients from the technique of [15], for the primal version of bounding the number of $k$-sets in a set of $n$ points in $\mathbb{R}^{3}$. The upper bound established in [15] is $O\left(n k^{3 / 2}\right)$, and is thus better than the bound we obtain here, for the case of (planes and) pseudoplanes (see full version [17]). The main reason for following this weaker analysis is the availability of the result of Tamaki and Tokuyama [18] on 
diamond-free graphs in arrangements of pseudolines, which leads to an extended dual version of the Crossing Lemma. It is definitely an intriguing hopefully not too difficult, challenge to extend, to the case of pseudoplanes, a dual version of the sharper analysis in [15].

Another line of research is to relax one or more of properties (i)-(iv), defining a family of pseudoplanes, as described in Section 2, with the goal of extending our analysis and obtaining nontrivial bounds for the complexity of the $k$-level in arrangements of more general surfaces. Property (iv) seems to be the most restrictive property among the four, namely requiring the $x y$-projections of all intersection curves from $\Lambda$ to form a family of pseudolines in the plane (although it trivially holds for the case of planes). The main use of this property in our analysis is in proving a generalized dual version of the Lovász Lemma (Lemma 9), as it (a) facilitates the applicability of topological sweeping, and (b) allows us to exploit the extended notion of antipodality, as in Lemma 7. It is an interesting challenge to find refined techniques that can extend this analysis to situations where the arrangement within the curtain is not an arrangement of pseudolines. One open direction is to find a different proof technique of the Lovász Lemma that is not based on sweeping. This would also be very interesting for the original case of planes (or of lines in the plane).

In studying the complexity of a level in an arrangement of more general surfaces, how far can we relax the constraints that these surfaces must satisfy in order to enable us to obtain sharp (significantly subcubic) bounds on the complexity of a level?

Finally, can our technique be extended to higher dimensions? For example, can we obtain a sharp bound in four dimensions, similar to the bound in Sharir [14] for $k$-sets in $\mathbb{R}^{4}$ ?

\section{References}

1 P. K. Agarwal, B. Aronov, T. M. Chan, and M. Sharir. On levels in arrangements of lines, segments, planes, and triangles. Discrete Comput. Geom., 19:315-331, 1998.

2 P. K. Agarwal and M. Sharir. Davenport-Schinzel Sequences and Their Geometric Applications, pages 216-217. Cambridge University Press, NY, USA, 1995.

3 P. K. Agarwal and M. Sharir. Pseudo-line arrangements: duality, algorithms, and appliations. SIAM J. Comput., pages 34:526-552, 2005.

4 M. Ajtai, V. Chvátal, M. M. Newborn, and E. Szemerédi. Crossing-free subgraphs, pages 9-12. North-Holland Mathematics Studies, Amsterdam, 1982.

5 I. Bárány, Z. Füredi, and L. Lovász. On the number of halving planes. Combinatorica, 10:175-183, 1990.

6 T. M. Chan. On the bichromatic $k$-set problem. ACM Transactions on Algorithms, 6(4):62:162:20, 2010.

7 T. M. Chan. On levels in arrangements of surfaces in three dimensions. Discrete Comput. Geom., 48:1-18, 2012.

8 T. K. Dey. Improved bounds on planar $k$-sets and related problems. Discrete Comput. Geom., 19:373-382, 1998.

9 T. K. Dey and H. Edelsbrunner. Counting triangle crossing and halving planes. Discrete Comput. Geom., 12:281-289, 1994.

10 H. Edelsbrunner. Algorithms in Combinatorial Geometry, pages 271-291. Springer Verlag, Heidelberg, 1987.

11 H. Edelsbrunner and L. Guibas. Topologically sweeping an arrangement. J. Computer Systems Sci., 38:165-194, 1989 .

12 J. Hershberger and J. Snoeyink. Sweeping arrangements of curves. Proc. 5th ACM Sympos. on Computational Geometry, pages 354-363, 1989.

13 G. Nivasch. An improved, simple construction of many halving edges. Contemporary Mathematics, 453:299-306, 2008. 
14 M. Sharir. An improved bound for $k$-sets in four dimensions. Combinatorics, Probability and Computing, 20:119-129, 2011.

15 M. Sharir, S. Smorodinsky, and G. Tardos. An improved bound for $k$-sets in three dimensions. Discrete Comput. Geom., 26:195-204, 2001.

16 M. Sharir and J. Zahl. Cutting algebraic curves into pseudo-segments and applications. $J$. Combinat. Theory, Ser. A, pages 150:37-42, 2017.

17 M. Sharir and C. Ziv. On the complexity of the $k$-level in arrangements of pseudoplanes, 2019. arXiv: 1903.07196.

18 H. Tamaki and T. Tokuyama. A characterization of planar graphs by pseudo-line arrangements. Algorithmica, 35:269-285, 2003.

19 G. Tóth. Point sets with many k-sets. Proc. 16th Annual Sympos. on Computational Geometry, 35:37-42, 2000.

20 C. Ziv. On the Complexity of the $k$-Level in Arrangements of Pseudoplanes. Master's thesis, School of Computer Science, Tel Aviv University, 2019. 